SCIENCE CHINA

Physics, Mechanics \& Astronomy

\title{
Quest towards ultimate performance in superconducting nanowire single photon detectors
}

\author{
Shigehito Miki* \\ Advanced ICT Research Institute, National Institute of Information and Communications Technology, Kobe, Hyogo 651-2492, Japan
}

Received November 1, 2017; accepted November 14, 2017; published online December 18, 2017

$\begin{array}{ll}\text { Citation: } & \text { S. Miki, Quest towards ultimate performance in superconducting nanowire single photon detectors, Sci. China-Phys. Mech. Astron. 61, 020331 (2018), } \\ \text { https://doi.org/10.1007/s11433-017-9134-8 }\end{array}$

In 2007, superconducting nanowire single photon detectors (SSPD or SNSPD) [1] made an outstanding impact in the field of quantum information technology by demonstrating quantum key distribution (QKD) over a 200-km optical fiber with a 42-dB optical loss using a practical SNSPD system [2]. This successful demonstration was realized thanks to its extremely low dark count rate (DCR) of a few $\mathrm{Hz}$ and short timing jitter of $60 \mathrm{ps}$, while the system detection efficiency (SDE) showed a poor value of $0.7 \%$ at a wavelength of $1550 \mathrm{~nm}$. Afterwards, significant efforts have been devoted to the improvement of SDE in practical systems, and remarkable advancements have been continually achieved in the past decade. The SDE of SNSPD is primarily determined by the product of three factors: optical coupling efficiency $\left(\eta_{\mathrm{oc}}\right)$, absorption efficiency $\left(\eta_{\mathrm{abs}}\right)$, and intrinsic detection efficiency $\left(\eta_{\text {int }}\right)$. Therefore, the advancements to increase each factor cumulatively resulted in the improvement of SDE.

In the initial stage of the SNSPD research, the nanowire device consists only of an $\mathrm{NbN}$ superconducting nanowire on the substrate. In this case, the nanowire cannot absorb the incident photons efficiently, because the thickness of the superconducting nanowire is only few nanometers. To achieve high $\eta_{\text {abs, }}$ Rosfjord et al. [3] demonstrated an integrated $\lambda / 4$ optical-cavity structure on the superconducting nanowire, and this approach could be successfully adapted in practically available devices with relatively large sensitive area $[4,5]$. The optical cavity design has evolved through various ap-

*Corresponding author (email: s-miki@nict.go.jp) proaches aimed at obtaining higher $\eta_{\text {abs }}$ : double-side cavity for back side illumination [6,7], optimized optical cavity for front side illumination [8], and cavity with distribution Bragg reflector (DBR) [9-11]. It is to be noted that such interesting approaches also made additional attempts to obtain the flexible wavelength spectrum by employing nonperiodic DBR [12], and to reduce the polarization sensitivity by optimizing the optical cavity design [13].

Considerable attention has also been focused on obtaining an $\eta_{\text {int }}$ that is as high as possible. Among various approaches aimed at increasing $\eta_{\text {int }}$, exploration of the optimal superconducting material is one of the most interesting topic. SNSPD with various superconducting materials have demonstrated excellent SDEs exceeding $80 \%$ at a wavelength of $1550 \mathrm{~nm}$. For example, SNSPD with amorphous WSi nanowire showed the highest SDE of $93 \%$ thus far, with widely saturated bias current dependencies at an operation temperature of $120 \mathrm{mK}$ [8]. However, an operation temperature of $120 \mathrm{mK}$ is not favorable from a practical point of view, because a relatively large and complicated cryocooler system such as an adiabatic demagnetization (ADR) cryocooler or a ${ }_{3} \mathrm{He}$ cryocooler is required. Recently, a high SDE of $78 \%$ has been reported at an operation temperature of $2.5 \mathrm{~K}$, which can be cooled by the compact Gifford-McMahon (GM) cryocooler system, even though the widely saturated bias-current dependencies have been lost [14]. However, the NbN films are the original superconducting material used in SNSPD since its proposal due to their adequately high $T_{\mathrm{c}}(16 \mathrm{~K}$ at $\sim 100 \mathrm{~nm}$ thickness and 
7-10 $\mathrm{K}$ at a thickness of few nanometers) [15] to operate at $4 \mathrm{~K}$ and the short relaxation time of quasi-particles to achieve high speed operation. After many significant advancements, NbN-SNSPDs have succeeded in demonstrating high SDEs with the GM cryocooler system [7]. In particular, a manuscript reported by Zhang et al. [11] shows an important result of achievement of $\sim 90 \%$ SDE at a wavelength of $1550 \mathrm{~nm}$ by applying sophisticated technologies. Although the results showed a narrower saturated region in the bias-current dependencies than that of WSi-SNSPD operating at $120 \mathrm{mK}$, wider saturated bias-current dependency has been demonstrated in NbTiN-SNSPD by applying the avalanche switching architecture [16]. It should be noted that MoSi-SNSPD has also demonstrated a high SDE of $\sim 80 \%$, and therefore, can also serve as a prospective candidate [13].

Although the SDE of SNSPDs have reached the near-unity region as described above, a quest towards even further improvement in their performance is underway. Present SNSPDs with $\mathrm{NbN}$, WSi, and MoSi superconducting nanowires continue to suffer from several trade-off relationships in their specifications (operation temperature, SDE, and timing jitter, for example). In addition to the simultaneous achievement of high SDE, DCR, timing jitter, maximum speed, and broadband sensitivity, ensuring the usability in terms of factors such as operation temperature, size of cryocooler system, bias current margin, reliability, durability, etc., is crucial to the development and improvement of SNSPDs.

1 G. N. Gol'tsman, O. Okunev, G. Chulkova, A. Lipatov, A. Semenov,
K. Smirnov, B. Voronov, A. Dzardanov, C. Williams, and R. Sobolewski, Appl. Phys. Lett. 79, 705 (2001).

2 H. Takesue, S. W. Nam, Q. Zhang, R. H. Hadfield, T. Honjo, K. Tamaki, and Y. Yamamoto, Nat. Photon 1, 343 (2007), arXiv: 0706.0397.

3 K. M. Rosfjord, J. K. W. Yang, E. A. Dauler, A. J. Kerman, V. Anant, B. M. Voronov, G. N. Gol'Tsman, and K. K. Berggren, Opt. Express 14, 527 (2006).

4 X. Hu, T. Zhong, J. E. White, E. A. Dauler, F. Najafi, C. H. Herder, F. N. C. Wong, and K. K. Berggren, Opt. Lett. 34, 3607 (2009).

5 S. Miki, T. Yamashita, M. Fujiwara, M. Sasaki, and Z. Wang, Opt. Lett. 35, 2133 (2010), arXiv: 1004.0037.

6 D. Rosenberg, A. J. Kerman, R. J. Molnar, and E. A. Dauler, Opt. Express 21, 1440 (2013).

7 S. Miki, T. Yamashita, H. Terai, and Z. Wang, Opt. Express 21, 10208 (2013), arXiv: 1303.6381.

8 F. Marsili, V. B. Verma, J. A. Stern, S. Harrington, A. E. Lita, T. Gerrits, I. Vayshenker, B. Baek, M. D. Shaw, R. P. Mirin, and S. W. Nam, Nat. Photon 7, 210 (2013), arXiv: 1209.5774.

9 D. Liu, S. Miki, T. Yamashita, L. You, Z. Wang, and H. Terai, Opt. Express 22, 21167 (2014).

10 B. Baek, J. A. Stern, and S. W. Nam, Appl. Phys. Lett. 95, 191110 (2009).

11 W. J. Zhang, L. X. You, H. Li, J. Huang, C. L. Lv, L. Zhang, X. Y. Liu, J. J. Wu, Z. Wang, and X. M. Xie, Sci. China-Phys. Mech. Astron. 60, 120314 (2017).

12 T. Yamashita, K. Waki, S. Miki, R. A. Kirkwood, R. H. Hadfield, and H. Terai, Sci. Rep. 6, 35240 (2016).

13 V. B. Verma, B. Korzh, F. Bussières, R. D. Horansky, S. D. Dyer, A. E. Lita, I. Vayshenker, F. Marsili, M. D. Shaw, H. Zbinden, R. P. Mirin, and S. W. Nam, Opt. Express 23, 33792 (2015), arXiv: 1504.02793.

14 V. B. Verma, B. Korzh, F. Bussières, R. D. Horansky, A. E. Lita, F. Marsili, M. D. Shaw, H. Zbinden, R. P. Mirin, and S. W. Nam, Appl. Phys. Lett. 105, 122601 (2014), arXiv: 1406.1810.

15 Z. Wang, A. Kawakami, Y. Uzawa, and B. Komiyama, J. Appl. Phys. 79, 7837 (1996).

16 S. Miki, M. Yabuno, T. Yamashita, and H. Terai, Opt. Express 25, 6796 (2017), arXiv: 1701.07247. 